\title{
Ability of an ELISA-Based Seed Health Test to Detect Erwinia stewartii in Maize Seed Treated with Fungicides and Insecticides
}

\author{
J. K. Pataky, Department of Crop Sciences, University of Illinois, Urbana 61801; C. C. Block, United States De- \\ partment of Agriculture-Agricultural Research Service North Central Plant Introduction Station, Ames, IA 50011; \\ P. M. Michener, Department of Crop Sciences, University of Illinois; L. M. Shepherd and D. C. McGee, Depart- \\ ment of Plant Pathology and Seed Science Center, Iowa State University, Ames 50011; and D. G. White, Department \\ of Crop Sciences, University of Illinois
}

\begin{abstract}
Pataky, J. K., Block, C. C., Michener, P. M., Shepherd, L. M., McGee, D. C., and White, D. G. 2004. Ability of an ELISA-based seed health test to detect Erwinia stewartii in maize seed treated with fungicides and insecticides. Plant Dis. 88:633-640.

Two sets of experiments were done to examine whether seed-treatment chemicals affected the ability of an enzyme-linked immunosorbent assay (ELISA)-based seed health test to detect Erwinia stewartii. The chemicals evaluated included Actellic, Apron, Captan, Cruiser, Gaucho, Maxim, Poncho, Thiram, and Vitavax in 11 seed-treatment combinations. In one experiment, seed-treatment chemicals were evaluated quantitatively in a critical region of ELISA absorbance values near 0.5 using maize seed that were spiked with uniform quantities of a liquid suspension of E. stewartii. The number of bacteria in each sample was estimated from ELISA absorbance values using standard curves. Log CFU of E. stewartii per sample were not significantly different among the untreated control and the 11 seed treatments compared with Tukey's Studentized Range Test $(P=0.05)$. Means of $\log \mathrm{CFU} / \mathrm{ml}$ for all treatments were tightly clustered around 5.70 which corresponded to an absorbance value of 0.440 and a bacterial population of about $500,000 \mathrm{CFU} / \mathrm{ml}$. In a second set of experiments, seed treatment chemicals were evaluated based on qualitative decisions that resulted from the ELISA-based seed health test of seed lots of Jubilee and A632 infected with E. stewartii. The number of negative samples was not substantially greater than expected based on binomial probabilities except for samples of Captan/Vitavaxtreated A632, which we considered to be a type I error. The mean absorbance values of positive samples ranged from 1.42 to 1.72 for A632 and from 1.51 to 1.91 for Jubilee and did not differ significantly among the seed treatments. There was no consistent evidence from these experiments that fungicide or insecticide seed treatments interfered with the sensitivity of the ELISA or altered low (e.g., 0.5) or high (e.g. 1.4 to 1.9) absorbance values. The ability of the ELISAbased seed health test to detect E. stewartii in maize seed was not affected by these seed treatments.
\end{abstract}

Many countries prohibit or place restrictions on importation of maize seed produced in the United States in order to prevent the introduction of Erwinia stewartii (Smith) Dye (Syn. Pantoea stewartii), which causes Stewart's bacterial wilt. These phytosanitary restrictions are widespread even though the results of several recent studies demonstrate that the risk of introducing E. stewartii on seed is exceedingly low if good-quality seed is produced

Corresponding author: J. K. Pataky

E-mail: j-pataky@uiuc.edu

Partial funding for this research provided by Pioneer Hi-Bred International, Johnston, IA.

Mention of a trademark or proprietary product does not constitute a guarantee or warranty of the product by the USDA and does not imply its approval to the exclusion of other products that may also be suitable.

Accepted for publication 21 January 2004.

Publication no. D-2004-0311-02R

(C) 2004 The American Phytopathological Society health testing for E. stewartii in the United States. Results from the ELISA used in the seed health test are interpreted qualitatively (i.e., the pathogen is determined to be present or absent in a sample). In spite of this qualitative interpretation of results, absorbance values from the ELISA for $E$. stewartii form an S-shaped curve when plotted against the number of bacteria in a sample well $(1,9$, C. C. Block and L. M. Shepherd, unpublished). At the low end of the curve, bacteria are not present in the sample or the number of bacteria is below the detection threshold of the assay (e.g., $10^{5}$ cells $/ \mathrm{ml}$ ). At the high end, the signal is completely saturated by a large number of bacteria in the sample. Heavily infected seed lots produce strong absorbance responses that are easy to detect. The problematic seed lots are those that produce a weak absorbance signal, in the range of 0.1 to 0.5 , near the threshold value of positive samples.

The establishment of the positive threshold is based on a subjective decision. Sutula et al. (14) reported the results of a survey of 81 research papers in which ELISA threshold values were established to determine samples that were considered positive. Two times the mean absorbance value of the negative controls was the most frequent of five methods used to establish a positive threshold value. The other four methods included three times the negative mean, and the negative mean plus two, three, or four standard deviations above the negative mean. Because of the subjective nature of positive thresholds, some laboratories, such as Agdia, include a third category of samples termed "elevated". Elevated samples are those that test below the subjective positive threshold, but possess an optical density value that is above the negative control. Thus, these samples may need to be retested or resampled. Regardless of the method used to establish threshold values for positive samples, any factor that masks or inhibits an ELISA signal may lower an absorbance value below the minimums that are considered positive or elevated.

Although there is no evidence that seedtreatment chemicals alter the sensitivity of the ELISA for E. stewartii, regulatory agencies have been cautious by requiring that maize seed submitted for Stewart's 
wilt phytosanitary tests not be treated with seed-treatment chemicals. If untreated samples from each seed lot are not preserved, seed cannot be submitted for Stewart's wilt phytosanitary tests or exported to countries requiring those tests. Maize seed often is treated with one or more fungicides, insecticides, or both; therefore, it would be useful to know if those chemicals affect the sensitivity of the ELISA for E. stewartii.

The objective of this study was to evaluate the ability of the ELISA-based seed health test to detect E. stewartii in maize seed treated with commonly used fungicides or insecticides.

\section{MATERIALS AND METHODS}

Two sets of experiments were done to examine whether seed-treatment chemicals affected the ability of the ELISA to detect E. stewartii. In one set of experiments, the effects of seed-treatment chemicals were evaluated based on qualitative decisions resulting from the ELISA-based seed health test. In these experiments, chemically treated and nontreated seed infected with E. stewartii were compared based on the number of samples that were classified as E. stewartii negative from the ELISAbased seed health test. In a second experiment, the effects of seed-treatment chemicals were evaluated quantitatively by spiking treated but uninfected maize seed with uniform aliquots of E. stewartii and then testing these samples by ELISA to determine if any of the chemicals affected the sensitivity of the assay.
Seed treatments. In both experiments, seed were treated at the manufacturer's recommended rates with 1 of 11 combinations of commonly used fungicide or insecticide seed treatments (Table 1). Nontreated seed also were included as a control treatment. All seed treatments were applied by personnel at Pioneer Hi-Bred International, Johnston, IA. Application rates were: Actellic (pirimiphos-methyl), $1.3 \mathrm{ml} / 100 \mathrm{~kg}$ of grain; Apron (mefanoxam), 1.0 a.i./100 kg of seed; Captan 400C (captan), $75 \mathrm{~g}$ a.i./100 kg of seed; Cruiser 5 FS (thiamethoxam), $1.25 \mathrm{mg}$ a.i./kernel; Gaucho 600 (imidacloprid), $1.25 \mathrm{mg}$ a.i./kernel; Maxim (fludioxonil), $2.5 \mathrm{~g}$ a.i./100 kg of seed; Poncho 600 (clothianidin), $1.25 \mathrm{mg}$ a.i./kernel; Thiram (thiram), $94 \mathrm{~g}$ a.i./100 kg of seed; and Vitavax (carboxin), $78 \mathrm{~g}$ a.i./100 kg of seed.

The seed treatments were applied to groups of about 4,000 kernels of E. stewartii-infected seed of the sweet corn hybrid Jubilee and the field corn inbred A632 that were assayed in the first set of experiments. The seed treatments also were applied to noninfected (i.e., "healthy") seed of the Pioneer Brand hybrid 33H67 that was produced in an area where Stewart's wilt did not occur. Treated seed of Pioneer Brand hybrid 33H67 were used in both sets of experiments (i.e., the qualitative trials of infected seed and the quantitative trials of spiked samples).

Qualitative decisions from the ELISA-based seed health tests of infected seed. In this experiment, the effects of seed-treatment chemicals were evaluated based on qualitative decisions (i.e., negative or positive samples) made from the ELISA-based seed health test. Seed infected with E. stewartii were produced on Jubilee and on A632. Infected seed lots were assayed to estimate the percentage of infected kernels. Seed-treatment chemicals were applied to infected seed lots and to healthy seed of Pioneer Brand hybrid $33 \mathrm{H} 67$ as described above. Seed from infected and healthy seed lots were blended together on a 100-kernel sample basis to produce samples that had approximately a $90 \%$ probability of containing an infected kernel. The ELISA-based seed health test then was used to assay samples for E. stewartii. A total of 66 samples were assayed independently at the University of Illinois or the Iowa State University Seed Science Center for each of the 11 seed treatments and the nontreated control (726 total). The effects of seed treatments were tested statistically based on the expected probability of negative samples based on binomial distributions.

Production of seed infected with $E$. stewartii. Seed infected with E. stewartii was produced on Jubilee, a sweet corn hybrid that is highly susceptible to Stewart's wilt, and on A632, a susceptible dent corn inbred. Infection of Jubilee and A632 frequently is systemic and symptoms of Stewart's wilt are distinct on these maize lines. Jubilee plants were inoculated to ensure that kernels would be E. stewartii infected. Plants of A632 were infected

Table 1. Negative, positive, and elevated outcomes and mean absorbance values of positive outcomes when samples of infected seed of A632 and Jubilee that had been treated with fungicides, insecticides, or both were tested for Erwinia stewartii using an enzyme-linked immunosorbent assay (ELISA)-based seed health test ${ }^{\mathrm{u}}$

\begin{tabular}{|c|c|c|c|c|c|c|c|c|}
\hline \multirow[b]{2}{*}{ Treatment } & \multicolumn{4}{|c|}{ A632 } & \multicolumn{4}{|c|}{ Jubilee } \\
\hline & Negative $^{v}$ & Elevated $^{w}$ & Positive $^{x}$ & Meany & Negative $^{v}$ & Elevated $^{w}$ & Positive $^{x}$ & Mean $^{y}$ \\
\hline Nontreated & 4 & 0 & 29 & 1.52 & 0 & 0 & 33 & 1.51 \\
\hline Captan/Apron & 5 & 3 & 25 & 1.43 & 0 & 0 & 33 & 1.91 \\
\hline Captan/Vitavax & $10 * *$ & 2 & 21 & 1.46 & 0 & 0 & 33 & 1.71 \\
\hline Captan/Vitavax/Apron & 5 & 1 & 27 & 1.42 & 0 & 0 & 33 & 1.69 \\
\hline Captan/Vitavax/Thiram & 5 & 1 & 27 & 1.49 & 1 & 0 & 32 & 1.75 \\
\hline Cruiser & 4 & 0 & 29 & 1.64 & 0 & 0 & 33 & 1.74 \\
\hline Maxim XL & $6^{*}$ & 1 & 26 & 1.72 & 2 & 0 & 31 & 1.62 \\
\hline Maxim/Apron & 2 & 1 & 30 & 1.42 & 0 & 0 & 33 & 1.66 \\
\hline Maxim/Apron/Gaucho & 5 & 1 & 27 & 1.49 & 0 & 0 & 33 & 1.79 \\
\hline Poncho & $7 *$ & 0 & 26 & 1.53 & 0 & 0 & 33 & 1.80 \\
\hline Thiram & 5 & 0 & 28 & 1.51 & 2 & 0 & 31 & 1.56 \\
\hline Thiram/Actellic & 4 & 2 & 27 & 1.55 & 0 & 0 & 33 & 1.66 \\
\hline TOTAL & 62 & 12 & 322 & ns & 5 & 0 & 391 & $\mathrm{~ns}$ \\
\hline Critical value $(P=0.97)^{\mathrm{z}}$ & $>5$ & $\ldots$ & $\ldots$ & $\ldots$ & $>3$ & $\ldots$ & $\ldots$ & $\ldots$ \\
\hline
\end{tabular}

u The number of negative samples is greater than expected $(P<0.03)$ based on binomial distributions when 5.7\% (**) or $7.0 \%$ (*) of A632 kernels were $E$. stewartii infected; $\mathrm{ns}=$ treatment means not significantly different $(P=0.05)$ when compared by analysis of variance. Waller-Duncan Bayesian least significant difference values $(k=100)$ for separation of treatment means were 0.595 for A632 and 0.379 for Jubilee.

${ }^{v}$ Negative outcomes recorded when absorbance was within two times (e.g., $<0.13$ ) of the mean absorbance value of all negative controls on a specific ELISA plate.

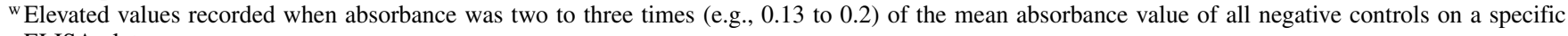
ELISA plate.

${ }^{x}$ Positive outcomes recorded when absorbance value was above three times (e.g., $\left.>0.2\right)$ of the mean absorbance value of all negative controls on a specific ELISA plate.

y Mean absorbance value for all positive outcomes.

${ }^{\mathrm{z}}$ Critical value $=$ number of negative samples for each treatment that results in rejection at the 0.03 level of the null hypothesis that the proportion of negative and positive samples are similar to those expected from the binomial distribution where $n=33$ and $P=0.9635$ for Jubilee (estimated proportion of positive 25-kernel samples of Jubilee with $12.4 \%$ kernel infection) and $n=33$ and $P=0.8822$ for A632 (estimated proportion of positive 36-kernel samples of A632 with 5.7\% kernel infection). 
naturally. Thus, the A632 seed lot was expected to have a more varied assortment of infected kernels (i.e., greater variation in number of bacteria per kernel) and was expected to produce a wider range of absorbance values from the ELISA.

About 0.02 ha of Jubilee was planted 19 May 2000 at the University of Illinois South Farms, Urbana. Standard production practices were followed. Pollination was not controlled. Therefore, kernels of Jubilee assayed in these experiments were produced on open-pollinated $F_{1}$ hybrids. Plants were inoculated five times at 2- to 3-day intervals beginning 2 weeks after pollination. E. stewartii inoculum $(3 \mathrm{ml})$ was injected into shanks of the primary ears of each plant using a modified hog vaccinator. Inocula were produced from a mixture of naturally occurring E. stewartii isolates collected from southern Illinois in the spring and isolates collected from Urbana, IL the previous fall. Isolates collected in the fall were maintained as a bulked population on infected plants in a greenhouse. To produce inocula, symptomatic leaves were surface sterilized in $95 \%$ ethanol for $10 \mathrm{~s}$ and rinsed twice in deionized water. Twenty $1.5-\mathrm{cm}^{2}$ pieces of leaf tissue were cut from symptomatic areas and placed in 1.5 liters of nutrient broth. Inocula were incubated on benchtop shakers at room temperature overnight.

Primary ears were harvested approximately 40 days after pollination and dried at ambient air temperatures to $15 \%$ moisture. Seed were shelled from ears, bulked, and stored in a refrigerated seed storage room. Approximately $40 \mathrm{~kg}$ of seed were produced.

Seed of the dent corn inbred A632 were harvested from a 0.01-ha plot at Ames, IA where plants were infected naturally by $E$. stewartii. The plot was planted 13 May 1992 and flea beetles were first observed 6 July. Ears were harvested 24 September from plants identified earlier in the season with symptoms of systemic Stewart's wilt. Seed were dried at $32^{\circ} \mathrm{C}$ to $11 \%$ moisture, shelled from ears, bulked, and stored at $5^{\circ} \mathrm{C}$ in a seed storage room.

Estimates of percentage of $E$. stewartii-infected kernels. The percentage of kernels infected with $E$. stewartii was estimated initially from samples of untreated seed from the two seed lots (Jubilee and A632) using a commercially available ELISA reagent set (Agdia, Inc., Elkhart, IN) that is sensitive to about $10^{5} \mathrm{CFU} / \mathrm{ml}$. The ELISA procedure has been described previously $(9,11)$. Seed of Jubilee were assayed individually and as five-kernel samples. A total of 736 kernels were selected randomly and assayed 3 or 7 months after harvest (i.e., 368 kernels assayed each time). Also, 716 five-kernel samples were selected and assayed on two consecutive days about 4 months after harvest. Estimates of the percentage of kernels infected with E. stewartii were determined from samples of individual kernels. Estimates also were determined from five-kernel samples using a formula for estimating proportions from group samples (7): $p=1$ $-(1-y / n)^{1 / \mathrm{k}}$, where $p=$ the proportion of E. stewartii-infected kernels, $y=$ the number of positive samples, $n=716$ (the total number of samples assayed), and $k=5$ (the number of kernels assayed in each sample that potentially were E. stewartii infected). A $95 \%$ confidence interval was calculated for each estimate. For the A632 seed lot, the estimate of infected kernels and confidence interval were calculated from assays of 685 individual kernels.

A second set of estimates of the percentage of infected kernels was derived from the number of E. stewartii-positive outcomes from the ELISA-based seed health tests of all 396 samples (33 samples of 12 treatments) of Jubilee and A632 as described below. Infected kernels were estimated from the formula for group samples (7) with $n=$ 396 and $k=25$ for Jubilee and $k=36$ for A632. An approximate $95 \%$ confidence interval was calculated for each point estimate using the SeedCalc5 spreadsheet.

Samples for the ELISA-based seed health test. The ELISA-based seed health test for E. stewartii developed by the Iowa State University Seed Science Center (D. McGee, unpublished) uses 100-kernel samples. In this experiment, the effects of seed treatments could not be tested effectively if 100-kernel samples of the original E. stewartii-infected Jubilee and A632 seed lots were assayed because all samples were likely to test positive based on binomial sampling probabilities (i.e., the probability of a 100-kernel sample of Jubilee or A632 containing zero E. stewartii-infected kernels was extremely unlikely). Therefore, treated seed from the E. stewartiiinfected seed lots of Jubilee and A632 were blended, on a per sample basis, with healthy seed of Pioneer hybrid 33H67 that received the same seed treatments. For Jubilee, each 100-kernel sample consisted of 75 kernels of healthy Pioneer hybrid 33 H67 and 25 kernels of Jubilee. The initial estimate of the percentage of infected Jubilee kernels was about $12.4 \%$ (see Results); thus, samples were expected to contain an average of 3.1 infected kernels of Jubilee. Based on probabilities from the binomial distribution (Fig. 1), there was about a $3.6 \%$ chance that any given sample contained no $E$. stewartii-infected kernels of Jubilee (i.e., $P_{(\mathrm{S}=0)}=0.036$ when $p=$ 0.124 and $n=25$ ). For A632, each $100-$ kernel sample consisted of 64 kernels of healthy Pioneer hybrid 33H67 and 36 kernels of A632. The initial estimate of the percentage of infected A632 kernels was $7.0 \%$ (see Results); thus, samples were expected to contain an average of 2.52 infected kernels of A632. Based on probabilities from the binomial distribution (Fig. 2), there was a $7.3 \%$ chance that any given sample contained no E. stewartiiinfected kernels of A632 (i.e., $P_{(\mathrm{S}=0)}=$ 0.073 when $p=0.07$ and $n=36$ ). Binomial distributions for the number of infected kernels per sample also were calculated for

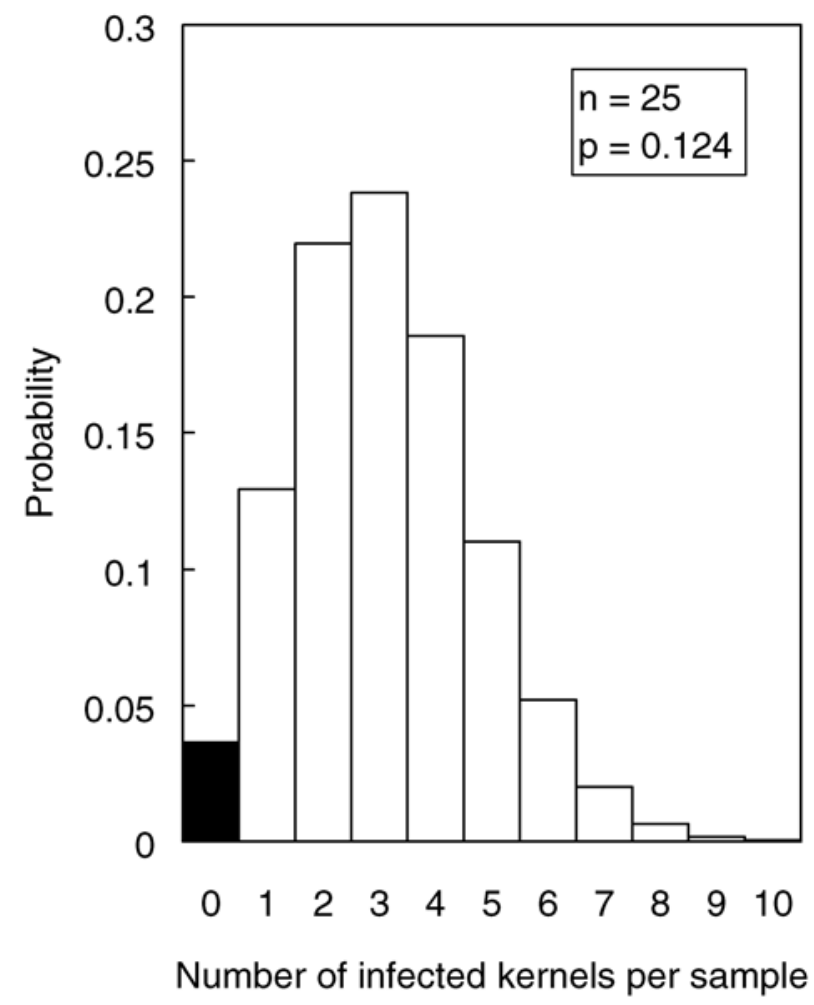

Fig. 1. Probability of 0 to 10 Erwinia stewartii-infected kernels in a 25-kernel sample of seed of Jubilee for which the incidence of kernel infection is $12.4 \%$. The probability of a sample having no infected kernels is about 0.036 (shaded area). 
A632 using 5.7\% as the estimate of kernel infection because several of the A632infected kernels had weak ELISA signals when tested individually (see Results). There was some concern that, if one kernel with only a few bacteria was the only infected kernel present in a 100-kernel sam- ple, the absorbance signal might be lost due to dilution. When the estimate of kernel infection was $5.7 \%$, there was an $11.6 \%$ chance that any given sample contained no E. stewartii-infected kernels of A632 (i.e., $P_{(\mathrm{S}=0)}=0.116$ when $p=0.057$ and $n=36)$.

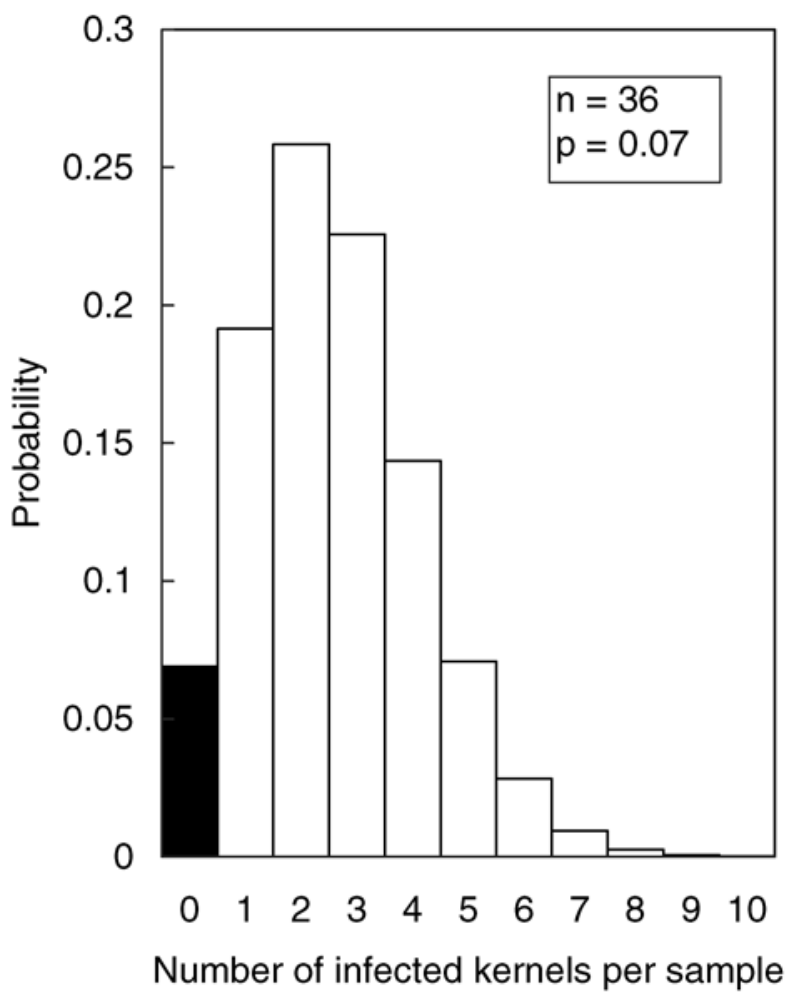

Fig. 2. Probability of 0 to 10 Erwinia stewartii-infected kernels in a 36-kernel sample of seed of A632 for which the incidence of kernel infection is $7.0 \%$. The probability of a sample having no infected kernels is about 0.073 (shaded area).

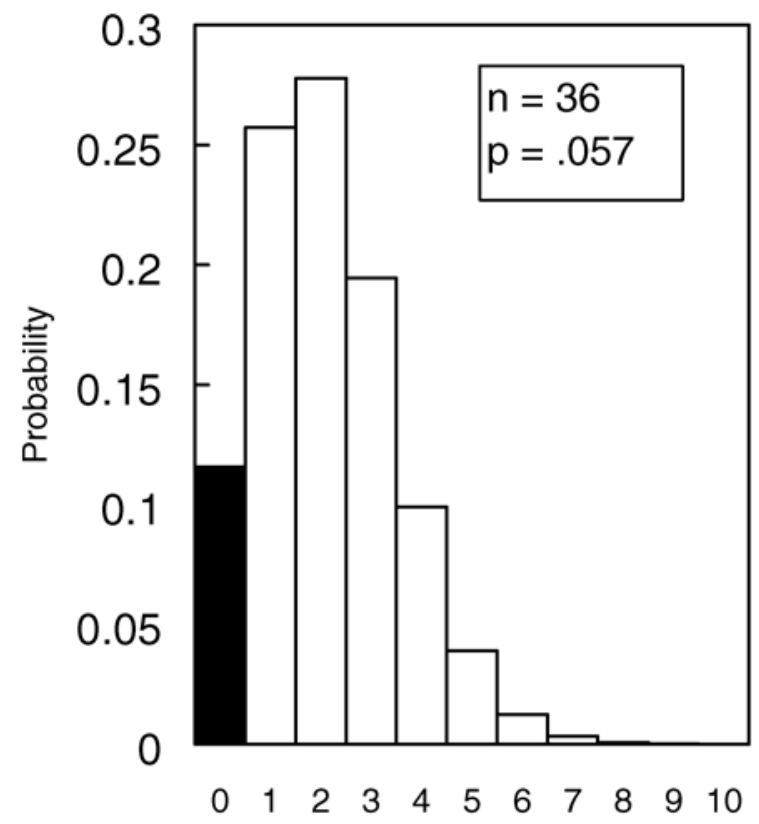

\section{Number of infected kernels per sample}

Fig. 3. Probability of 0 to 10 Erwinia stewartii-infected kernels in a 36-kernel sample of seed of A632 for which the incidence of kernel infection is $5.7 \%$. The probability of a sample having no infected kernels is about 0.121 (shaded area).

ELISA-based seed health test of infected seed. Samples were soaked overnight in a sample extraction buffer of 100 $\mathrm{ml}$ of phosphate-buffered saline (PBS) and $2 \mathrm{ml}$ of Tween-20. Samples were ground in a blender to fine particles and strained through two layers of cheesecloth. The filtrate then was assayed following the ELISA procedures described previously $(9,11)$. Two $100-\mu \mathrm{l}$ aliquots were taken from each sample and pipetted into two separate ELISA plate wells. Each 96-well ELISA plate contained three complete replicates of the 12 treatments (72 wells), one replicate of the 12 treatments for which the duplicate subsamples (second wells) were assayed on a second ELISA plate (12 wells), 2 wells each of a negative and a positive control (i.e., controls from the Agdia kit), and 2 wells each of four 100-kernel samples of chemically treated, healthy Pioneer hybrid $33 \mathrm{H} 67$ with treatments selected at random or wells to which PBS was added (i.e., PBS buffer control). Two ELISA plates were run at the same time to accommodate the replicate for which the duplicated samples were split between plates. For each plate, absorbance values of negative samples were calculated as the mean of the four treated, healthy samples, the PBS buffer control, and the negative control. Samples were considered negative for E. stewartii on a plate-by-plate basis if absorbance values were within two times the mean value of the negative control samples, which usually was near 0.06 . Samples were considered positive for $E$. stewartii if absorbance values were three times greater than the mean value of the negative control samples. Samples for which absorbance was between two and three times of the negative control were classified as having elevated values, but were considered to be positive samples in the analysis of binomial data.

Analysis of data from the ELISAbased seed health test of infected kernels. Absorbance values from all positive samples of the 11 seed treatments and the nontreated control were subjected to an analysis of variance (ANOVA) with treatment means compared using Waller-Duncan Bayesian least significant difference (BLSD) values $(k=100)$. Absorbance values from all samples also were grouped by intervals of 0.40 and their distributions were compared with the theoretical distributions of the number of infected kernels expected from 25-kernel samples of Jubilee and 36-kernel samples of A632 based on binomial probabilities (Figs. 1-3).

The effects of seed treatments on the ability of the ELISA-based seed health test to detect E. stewartii was tested with a binomial hypothesis test that compared the number of positive and negative samples for each treatment with the expected number of positive and negative outcomes based on binomial distributions. Thirtythree samples from both of the infected 
seed lots (i.e., Jubilee and A632) were assayed by ELISA for each of the 11 seed treatments and the nontreated control. Probabilities of 0 to 33 E. stewartiipositive outcomes from the ELISA-based seed health test were calculated from the binomial distribution. For samples that included 75 kernels of noninfected Pioneer hybrid $33 \mathrm{H} 67$ and 25 kernels of Jubilee, there was a 0.97 probability that three or fewer samples would be negative (i.e., contain no infected kernels). Thus, the null hypothesis would be rejected at $\alpha=0.03$ if 4 or more of the 33 samples of a specific seed treatment were negative. For samples that included 64 kernels of noninfected Pioneer hybrid $33 \mathrm{H} 67$ and 36 kernels of A632, there was a 0.97 probability that five or fewer samples would be negative. Thus, the null hypothesis would be rejected at $\alpha$ $=0.03$ if 6 or more of the 33 samples of a specific seed treatment were negative.

ELISA-based seed health test of spiked samples. In this experiment, the effects of seed-treatment chemicals were evaluated quantitatively by comparing ELISA absorbance values obtained from healthy treated seed samples that were spiked with uniform aliquots of an $E$. stewartii suspension. By assaying E. stewartii-spiked samples, the most critical range of absorbance values (i.e., 0.1 to 0.5 ) was examined more closely and sample-tosample variation was reduced substantially compared with the qualitative evaluations of infected seed.

Fifty-kernel samples of Pioneer hybrid $33 \mathrm{H} 67$, representing each of the 11 treatments and a nontreated control, were soaked overnight in $50 \mathrm{ml}$ of Agdia general extraction buffer at $5^{\circ} \mathrm{C}$, then ground in a commercial blender. A concentrated suspension of E. stewartii was prepared from a 48-h-old culture grown on nutrient broth yeast extract (NBY) agar and mixed in 15 $\mathrm{ml}$ of $0.85 \%$ saline. The suspension was adjusted to an absorbance (turbidity) of 0.400 using a spectrophotometer set at 590 $\mathrm{nm}$. Serial 10-fold dilutions of the concentrate were plated in a dilution series, in triplicate, on NBY agar to verify the bacterial concentration. Sodium azide then was added to the suspension to a level of $0.1 \%$ to inhibit further bacterial multiplication. The concentrate was diluted 1:1 with $0.85 \%$ saline and $0.2 \mathrm{ml}$ was added to each sample of ground corn (except for the uninfected controls) and mixed thoroughly. Estimates from a previously constructed standard curve of spectrophotometer absorbance versus bacterial concentration indicated that $0.2 \mathrm{ml}$ should give a moderate ELISA signal corresponding to that produced from a bacterial population of 500,000 to $800,000 \mathrm{CFU} / \mathrm{ml}$. After 20 to $30 \mathrm{~min}$, the samples were mixed again and allowed to settle for $15 \mathrm{~min}$. Two $100-\mu \mathrm{l}$ aliquots per sample were added to duplicate wells of an ELISA plate. Two replicates of the 11 seed treatments and nontreated control were placed on each ELISA plate in a randomized complete block arrangement. Two E. stewartiipositive and four negative buffer control wells were included on each plate. In the last 24 wells, a duplicate series of $12 \mathrm{E}$. stewartii standards was added so that a graph of absorbance values versus known bacterial concentration could be constructed from each ELISA plate. The standards were created by making a 1:9 dilution of the original bacterial concentrate, then making 12 doubling dilutions (i.e., $1: 2,1: 4,1: 8, \ldots 1: 2,048)$ in Agdia general extraction buffer. The quantity of bacteria in each standard was calculated later from the spectrophotometer standard curve. The range of standards spanned the absorbance range from close to the negative control to greater than 3.00.

Absorbance values for the standards ( $y$ axis) from each ELISA plate were plotted against $\log _{10}$-transformed values of the bacterial populations measured in CFU/ml ( $x$ axis). A four-parameter logistic equation was fitted to the standard ELISA absorbance curve for each plate using TableCurve 2D software (SPSS Inc., Chicago). All equations were fit to an $R^{2}$ value of 0.99 or higher. The resulting equation was used to estimate bacterial populations detected in each spiked seed sample based on its absorbance value. Twenty-six replicates (13 ELISA plates) were tested for each seed treatment. Populations were converted to $\log \mathrm{CFU} / \mathrm{ml}$ and treatment effects (i.e., the bacterial population estimated in each sample) were analyzed using the PROC GLM procedure in SAS (version 8.0; SAS Institute, Cary, NC). Treatments were compared using Tukey's Studentized range test.

\section{RESULTS}

Estimates of Jubilee and A632 kernels infected with $\boldsymbol{E}$. stewartii. Of 736 individual kernels of Jubilee assayed by ELISA, 92 were positive for E. stewartii. Of 368 kernels, 45 were positive from the assay done 3 months after harvest, and 47 of 368 kernels were positive 7 months after harvest. The mean percentage of E. stewartiiinfected kernels was estimated to be $12.5 \%$, with a $95 \%$ confidence interval of $\pm 2.4 \%$. When five-kernel samples of Jubilee were assayed by ELISA, 345 of 716 samples were positive for E. stewartii.
Based on estimates from group testing of binomial experiments, the mean percentage of E. stewartii-infected kernels was $12.3 \%$, with an approximate $95 \%$ confidence interval from 11.1 to $13.6 \%$. The point estimates from these two sets of assays were averaged to $12.4 \%$. In the assays of A632 seed, 48 of 685 individual kernels were positive for $E$. stewartii when tested by ELISA. The mean percentage of infected kernels of A632 was estimated to be $7.0 \%$, with a $95 \%$ confidence interval of $\pm 1.9 \%$. However, 9 of the 48 infected A632 kernels had weak ELISA absorbance signals in the range of 0.14 to 0.24 . These kernels might be classified as having elevated values and the weak absorbance signals might disappear if individual crushed kernels were suspended in $100 \mathrm{ml}$ of PBS rather than $2 \mathrm{ml}$ of buffer. If the nine kernels were not classified as positive samples, the mean percentage of infected kernels of A632 was estimated to be $5.7 \%$ with a $95 \%$ confidence interval of $\pm 1.7 \%$.

Over all 12 treatments of Jubilee assayed as part of the ELISA-based seed health test for treated seed, 391 of 39625 kernel samples (25 kernels of Jubilee blended with 75 kernels of healthy Pioneer hybrid 33H67) were positive for E. stewartii. Using the equation for group testing, the mean percentage of E. stewartiiinfected kernels was estimated to be $16 \%$, with an approximate $95 \%$ confidence interval from 13.1 to $19.7 \%$.

Over all 12 treatments of A632 assayed as part of the ELISA-based seed health test for treated seed, 322 of 396 36-kernel samples (36 kernels of A632 blended with 64 kernels of healthy Pioneer hybrid 33H67) were positive for E. stewartii, from which the mean percentage of $E$. stewartiiinfected kernels was estimated to be $4.6 \%$, with an approximate $95 \%$ confidence interval from 4.0 to $5.1 \%$. If the 12 samples of A632 with elevated absorbance values were included as positives, 334 of 396 36kernel samples were positive and the estimated percentage of E. stewartii-infected kernels was $5.1 \%$, with an approximate $95 \%$ confidence interval from 4.4 to $5.7 \%$.

Absorbance values from the ELISAbased seed health tests of infected seed. Absorbance values for duplicate samples of 23 negative controls ranged from 0.042 to 0.078 , with a mean value of 0.058 (Table 2). Absorbance values of 107 wells

Table 2. Absorbance values for negative and positive controls, wells to which phosphate buffer was added (PBST controls), and treated healthy seed assayed for Erwinia stewartii using an enzymelinked immunosorbent assay (ELISA)-based seed health test

\begin{tabular}{lcccc}
\hline & & \multicolumn{3}{c}{ Absorbance values $^{\mathbf{z}}$} \\
\cline { 3 - 5 } Treatment & $\boldsymbol{n}$ & Mean & Standard deviation & Range \\
\hline Negative control & 23 & 0.058 & 0.009 & $0.042-0.078$ \\
Positive control & 23 & 1.835 & 0.579 & $0.531-2.738$ \\
PBST control & 107 & 0.065 & 0.015 & $0.047-0.145$ \\
Treated healthy seed & 46 & 0.075 & 0.074 & $0.045-0.132$ \\
\hline
\end{tabular}

z Absorbance at $490 \mathrm{~nm}$. 
with PBS buffer and 46 duplicate samples of healthy treated seed were similar to the negative controls, except for 4 samples that had slightly elevated values from 0.13 to 0.145 (Tables 2 and 3). Absorbance values for duplicate samples of 23 positive controls ranged from 0.53 to 2.74 , with a mean of 1.84 (Table 2). All but one positive control had absorbance values above 1.0 (Table 3). Absorbance values for 792 samples of E. stewartii-infected seed lots (396 each of A632 and Jubilee) ranged from 0.052 to 3.0. Based on absorbance values below 0.13 (two times the negative control), 5 samples of Jubilee and 62 samples of A632 were negative for E. stewartii (Tables 1 and 3). Seed treatments were not a significant source of variation in the ANOVA of absorbance values from positive samples (i.e., Prob $>F=0.16$ for Jubilee and 0.77 for A632). The BLSD values for comparing mean absorbance values of positive samples among treatments were 0.595 for A632 and 0.379 for Jubilee (Table 1). Mean absorbance values of positive samples ranged from 1.42 to 1.72 for A632 and from 1.51 to 1.91 for Jubilee. The untreated control had the lowest mean absorbance value among the 12 treatments of Jubilee seed, but the value was not significantly different from any of the values from seed treated with chemicals.

Distributions of absorbance values of Jubilee and A632 were not similar to the theoretical distributions of number of infected kernels per sample (Table 3; Figs. 1-3). Nevertheless, the percentage of samples with absorbance values above 1.0 was only slightly lower than the theoretical percentage of samples with two or more infected kernels. In all, $78 \%$ of the samples of Jubilee (309 of 396) and 54\% of the samples of A632 (213 of 396) had absorbance values above 1.0 (Table 2). Based on the binomial distribution, $83 \%$ of the samples of Jubilee (with $12.4 \%$ kernel infection) and $62 \%$ of the samples of A632 (using the $5.7 \%$ estimate of infected kernels with strong ELISA signals) were expected to have two or more infected kernels per sample (Figs. 1 and 3). Similarly, the percentage of samples with absorbance values above 0.6 was $67 \%$ for A632; and, based on the binomial distribution, $73 \%$

Table 3. Distribution of absorbance values when positive and negative controls and infected seed of A632 and Jubilee were assayed for Erwinia stewartii using an enzyme-linked immunosorbent assay (ELISA)-based seed health test

\begin{tabular}{lcccccc}
\hline & \multicolumn{2}{c}{ Controls } & & \multicolumn{2}{c}{ E. stewartii-infected } \\
\cline { 2 - 3 } \cline { 5 - 6 } Range of absorbance values $^{\mathbf{y}}$ Negative $^{\mathbf{z}}$ & Positive & & $\mathbf{A 6 3 2}$ & Jubilee \\
\hline$<0.13$ & 171 & 0 & & 62 & 5 \\
$0.13-0.2$ & 4 & 0 & & 12 & 0 \\
$0.2-0.6$ & 0 & 1 & & 56 & 29 \\
$0.6-1.0$ & 0 & 0 & & 53 & 53 \\
$1.0-1.4$ & 0 & 6 & & 45 & 57 \\
$1.4-1.8$ & 0 & 3 & & 39 & 57 \\
$1.8-2.2$ & 0 & 3 & & 46 & 80 \\
$2.2-2.6$ & 0 & 9 & & 53 & 80 \\
$2.6-3.0$ & 0 & 1 & & 30 & 35
\end{tabular}

${ }_{\mathrm{y}}$ Absorbance at $490 \mathrm{~nm}$. Absorbance values below 0.13 were considered negative outcomes (samples free of E. stewartii), those between 0.13 and 0.20 were classified as elevated samples, and those above 0.20 were positive outcomes (samples containing $E$. stewartii-infected kernels).

${ }^{\mathrm{z}}$ Negative controls include wells to which phosphate-buffered saline plus Tween-20 were added, treated healthy seed, and negative controls from Agdia ELISA kits.

Table 4. Average $\log$ CFU of Erwinia stewartii per milliliter and size of bacterial populations estimated from absorbance values of enzyme-linked immunosorbent assay (ELISA) tests when known quantities of E. stewartii were added to samples of healthy maize seed treated with fungicides, insecticides, or both

\begin{tabular}{lccc}
\hline Treatment & Replicates & Average $\log$ CFU/ml & Estimated $_{\text {bacteria }}^{\mathbf{z}}$ \\
\hline Nontreated & 26 & $5.7096 \mathrm{a}$ & 512,400 \\
Captan/Apron & 26 & $5.6969 \mathrm{a}$ & 497,600 \\
Captan/Vitavax & 25 & $5.6980 \mathrm{a}$ & 498,900 \\
Captan/Vitavax/Apron & 25 & $5.7124 \mathrm{a}$ & 515,700 \\
Captan/Vitavax/Thiram & 26 & $5.6819 \mathrm{a}$ & 480,700 \\
Cruiser & 26 & $5.6962 \mathrm{a}$ & 496,800 \\
Maxim XL & 26 & $5.6962 \mathrm{a}$ & 496,800 \\
Maxim/Apron & 25 & $5.7032 \mathrm{a}$ & 504,900 \\
Maxim/Apron/Gaucho & 26 & $5.7015 \mathrm{a}$ & 502,900 \\
Poncho & 26 & $5.7012 \mathrm{a}$ & 502,600 \\
Thiram & 26 & $5.6888 \mathrm{a}$ & 488,400 \\
Thiram/Actellic & 26 & $5.6727 \mathrm{a}$ & 470,700 \\
\hline
\end{tabular}

${ }^{y}$ Log CFU/ml calculated from standard curves of absorbance values for each ELISA plate. Means followed by the same letter are not different. Minimum significant difference value is 0.0403 (Tukey's standardized range test, $P=0.05$ ).

${ }^{\mathrm{z}}$ Estimated bacterial population, converted from $\log \mathrm{CFU} / \mathrm{ml}$.
(288 of 396) of samples of A632 (using the $7.0 \%$ estimate of infected kernels with strong and weak ELISA signals) were expected to have two or more infected kernels.

Binomial hypothesis tests of the ability of the ELISA-based seed health test to detect $E$. stewartii from treated seed. For Jubilee seed treated with each of the 11 different seed treatments and the nontreated control, fewer than 3 of the 33 samples were negative for $E$. stewartii based on absorbance values below 0.13 (Table 1). Based on the binomial distribution, there was a $97 \%$ probability of finding 3 or fewer negative samples in each set of 33 samples. Therefore, the null hypothesis was not rejected for any of the 12 treatments because there was insufficient evidence to suggest that the seed treatments or the nontreated control affected the ability of the ELISA-based seed health to detect samples with E. stewartii-infected kernels of Jubilee.

For A632 seed treated with 8 of the 11 different seed treatments and the nontreated control, 5 or fewer of the 33 samples were negative for $E$. stewartii based on absorbance values below 0.13 (Table 1). Based on the binomial distribution, there was a $97 \%$ probability of finding 5 or fewer negative samples in each set of 33 samples. Therefore, the null hypothesis was not rejected because there was insufficient evidence to suggest that these eight seed treatments or the nontreated control affected the ability of the ELISA-based seed health to detect samples with E. stewartii-infected kernels of A632. There were 6, 7, and 10 negative samples of A632 seed treated with Maxim XL, Poncho, and Captan/Vitavax, respectively (Table 1). If $7 \%$ of the kernels of A632 were E. stewartii infected, the probability of having 6,7 , and 10 negative samples was $0.025,0.007$, and $6 \times 10^{-5}$ for the Maxim XL, TI-435, and Cap$\tan /$ Vitavax treated seed, respectively. In these cases, the null hypothesis was rejected because it was improbable to observe this number of negative outcomes from 33 36-kernel samples of seed with $7 \%$ E. stewartii-infected kernels. If the percentage of E. stewartii-infected kernels of A632 was $5.7 \%$ as estimated from individual kernels with strong ELISA signals, the probability of having 6,7 , and 10 negative samples was $0.20,0.10$, and 0.004 for Maxim XL, TI-435, and Captan/Vitavax treated seed. In that case, the null hypothesis was rejected with a low probability of a type I error $(P=0.004)$ only for the Cap$\tan /$ Vitavax treated seed.

ELISA-based seed health test of spiked samples. Log CFU of E. stewartii per milliliter did not differ among seed treated with the 11 combinations of fungicides or insecticides or the nontreated control when treatments were compared with Tukey's Studentized Range Test with a type I error rate of $P=0.05$ (Table 4) al- 
though the $F$ value of the ANOVA had an associated probability of 0.043 . The average $\log \mathrm{CFU} / \mathrm{ml}$ values for all treatments were tightly clustered around $\log 5.70$, corresponding to a bacterial population of about 500,000 CFU/ml (Table 4). There was no consistent ranking among seed treated with Maxim, Captan, Apron, Gaucho, Cruiser, TI-435, or Vitavax; however, the three seed treatments that contained Thiram (Thiram, Thiram/Vitavax/ Captan, and Thiram/Actellic) had the three lowest estimated bacterial populations even though the mean $\log \mathrm{CFU} / \mathrm{ml}$ of these treatments were not significantly different than the other treatments.

Treatments also did not differ $(P=$ $0.061)$ when the absorbance values from the ELISA were used in the ANOVA (data not shown) rather than $\log \mathrm{CFU} / \mathrm{ml}$. The average absorbance value for the untreated control was 0.451 . The mean absorbance values were lowest for the samples that contained Thiram (i.e., $0.434,0.426$, and 0.413 for Thiram, Thiram/Vitavax/Captan, and Thiram/Actellic, respectively).

\section{DISCUSSION}

Treating maize seed with fungicides, insecticides, or both did not significantly affect the ability of the ELISA-based seed health test to detect E. stewartii. Seed treatments did not interfere with the sensitivity of the ELISA by altering low (e.g., $<0.50$ ) or high (e.g., >1.0) absorbance values. It is unlikely that the seed treatment chemicals evaluated in these experiments would mask a weak ELISA signal by lowering the absorbance value, and thereby cause a weak positive sample to be interpreted as a negative sample. Similarly, seed treatments did not affect the number of positive and negative samples from the ELISA-based seed health test when compared with expected outcomes based on binomial distributions. Therefore, assays of treated seed appear to be as accurate as assays of nontreated seed.

For all treatments applied to E. stewartii-infected seed of Jubilee, the number of negative samples was within expectations of the null hypothesis derived from a binomial distribution of 25-kernel samples of seed with $12.4 \%$ E. stewartii-infected seed. For A632, the number of negative samples was within expectations of the null hypothesis for 36-kernel samples of seed with $5.7 \%$ infected-seed for all but one treatment, Captan/Vitavax $(P=0.004)$. When an estimate of 7\% infected A632 was used to calculate the distribution of number of infected kernels per sample, the null hypothesis was rejected for three treatments: Maxim XL $(P=0.025)$, Poncho $(P=0.007)$, and Captan/Vitavax $(P=$ $\left.6 \times 10^{-5}\right)$. Although there were more negative samples than expected for Cap$\tan$ /Vitavax-treated A632 (and possibly for Poncho- and Maxim XL-treated A632), it is not highly unusual to incorrectly reject the null hypothesis in 24 trials (i.e., 12 treatments $\times 2$ seed lots). Therefore, we believe that the larger than expected number of negative outcomes for Cap$\tan /$ Vitavax was a type I error. This interpretation is supported by the failure to reject the null hypothesis when Cap$\tan /$ Vitavax-treated seed of Jubilee were tested. It also is supported by the lack of differences among absorbance values of Captan/Vitavax-treated seed and other seed for the E-stewartii-positive samples of both A632 and Jubilee and for the E. stewartii-spiked samples of Pioneer hybrid $33 \mathrm{H} 67$.

There was some evidence that the strength of the ELISA signal (i.e., absorbance values) was associated with the number of E. stewartii-infected kernels per sample, although distributions of absorbance values from the ELISA-based seed health tests and theoretical distributions of the number of infected kernels per sample based on binomial probabilities were not strongly related. For both Jubilee and A632, the percentage of samples with absorbance values above 1.0 was only slightly lower (5 and 9\% lower for Jubilee and A632, respectively) than the expected number of samples with two or more infected kernels. Thus, we hypothesize that the majority of samples with strong ELISA signals probably had multiple infected kernels per sample. Although it cannot be confirmed from our data, samples with weaker signals may have been the result of one infected kernel per sample or fewer bacteria per multiple infected kernels. Interestingly, mean absorbance values of positive samples of A632 were lower than those for Jubilee except for untreated seed and seed treated with Maxim XL. Likewise, a greater number of samples of A632 than Jubilee were classified as elevated positives (absorbance values from 0.13 to 0.2 ) or had weak positive values from 0.2 to 0.6. Lower absorbance values for infected A632 may have been due to fewer infected kernels per sample (Figs. 1-3) or fewer bacteria per infected kernel than Jubilee. Two factors may have resulted in a greater number of bacteria per infected kernel of Jubilee. Jubilee is more susceptible to Stewart's wilt than A632, and seed of Jubilee were infected for these assays by injecting inocula in ear shanks. This procedure places a large number of bacteria directly at the base of the ear, potentially creating a high incidence of heavily infected kernels. The infected seed from the A632 seed lot resulted from natural systemic infection and the seed lot was not expected to contain as many heavily infected kernels.

Estimates of the percentage of E. stewartii-infected kernels of Jubilee and A632 differed slightly among the various assays. For Jubilee, point estimates from samples of individual kernels or five-kernel samples were extremely close, 12.5 and
$12.3 \%$, and confidence intervals overlapped, 10.1 to $14.9 \%$ and 11.1 to $13.6 \%$, respectively. Thus, it is reasonable to assume that percentage of infected Jubilee kernels was near $12.4 \%$, the value used to calculate binomial probabilities, or slightly higher.

For A632, the point estimate of infected kernels from 396 36-kernel samples of treated seed was $5.1 \%$, with a $95 \%$ confidence interval of 4.4 to $5.7 \%$. The point estimate from samples of individual kernels was $7 \pm 1.9 \%$; however, when kernels with weak ELISA signals (i.e., 0.14 to 0.2 , classified as elevated) were excluded as positives, the point estimate was $5.7 \pm$ $1.7 \%$. Although samples with elevated values usually are considered to be positive outcomes, it is reasonable to treat the elevated values from assays of individual kernels of A632 as negative outcomes because these samples were suspended in only $2 \mathrm{ml}$ of buffer. One might hypothesize that these samples with elevated values were due to kernels with too few bacteria to produce a strong signal. If these kernels were suspended in $100 \mathrm{ml}$ of buffer, as is used in the ELISA-based seed health test, it is likely that many would have been diluted below the $10^{5}$ cells $/ \mathrm{ml}$ detection threshold, particularly if only one infected kernel was present in the larger sample. Alternatively, samples with weak but elevated signals would be considered negative samples if the threshold value of the assay is subjectively set at three times the mean of the negative control rather than two times. Thus, classification of samples with absorbance values in the range of two to three times is not indisputable.

Although the classification of samples with weak ELISA signals may be of concern, seed responsible for these weak signals are extremely unlikely sources of $E$. stewartii transmission. Based on recent studies in which rates of seed-to-seedling transmission of E. stewartii were estimated to be near $0.06 \%(2,10)$, it seems unlikely that $E$. stewartii will be transmitted in seed that have a low percentage of infected kernels (e.g., 1\%). When seed were produced on plants inoculated with E. stewartii and 37 to $72 \%$ of the kernels were $E$. stewartii infected, Block et al. (2) observed 28 infected seedlings grown from 42,206 kernels $(0.066 \%)$ of seven different seed lots. In eight seed lots with less than $35 \%$ infected kernels, only a single incidence of seed-to-seedling transmission occurred in nearly 35,000 seedlings. Michener et al. (10) observed about $0.044 \%$ seed transmission among nearly 83,400 seedlings grown from a seed lot with $49 \%$ kernel infection, and they may have observed seed transmission in a seed lot with $16 \%$ kernel infection, although natural infection could not be excluded with a reasonable probability. However, Michener et al. (10) did not observe seed transmission from another seed lot with $12 \%$ kernel infection. 
Therefore, it seems that seed transmission is most likely to occur from seed lots with a high incidence of heavily infected seed that are detected easily in the ELISA-based seed health test due to strong ELISA signals. There should be little concern about seed transmission from samples with weak signals (i.e., two to three times the mean of negative controls) that are classified as elevated.

Seed-treatment chemicals would have to have a substantial effect on absorbance values in order to affect the phytosanitary value of the ELISA-based seed health test. There was no consistent evidence from these experiments that fungicide or insecticide seed treatments substantially affected the ability of the ELISA-based seed health test to detect $E$. stewartii in maize seed. Assays of treated seed appear to be no different than assays of nontreated seed.

\section{ACKNOWLEDGMENTS}

We thank J. Stevens, G. Lamka, and J. Callan (Pioneer HiBred International, Johnston, IA) for discussions about experimental procedures and assistance with seed treatments.

\section{LITERATURE CITED}

1. Blakemore, E. J. A., Law, J. R., and Reeves, J. C. 1999. PCR identification of Erwinia stewartii and its comparison with two other methods. Seed Sci. Technol. 27:385-396.

2. Block, C. C., Hill, J. H., and McGee, D. C. 1998. Seed transmission of Pantoea stewartii in field and sweet corn. Plant Dis. 82:775-780.

3. Block, C. C., Hill, J. H., and McGee, D. C. 1999. Relationship between late-season severity of Stewart's bacterial wilt and seed infection in maize. Plant Dis. 83:527-530.

4. Elliott, C., and Poos, F. W. 1934. Overwintering of Aplanobacter stewartii. Science 80:289290.

5. Elliott, C., and Poos, F. W. 1940. Seasonal development, insect vectors, and host range of bacterial wilt of sweet corn. J. Agric. Res. 60:645-686.

6. Esker, P. D., and Nutter, F. W., Jr. 2002. Assessing the risk of Stewart's disease of corn through improved knowledge of the role of the corn flea beetle vector. Phytopathology 92:668-670.

7. Hepworth, G. 1996. Exact confidence intervals for proportions estimated by group testing.
Biometrics 52:1134-1146.

8. Khan, A., Ries, S. M., and Pataky, J. K. 1996. Transmission of Erwinia stewartii through seed of resistant and susceptible field and sweet corn. Plant Dis. 80:398-403.

9. Lamka, G. L., Hill, J. D., McGee, D. C., and Braun, E. J. 1991. Development of an immunosorbent assay for seedborne Erwinia stewar tii in corn seeds. Phytopathology 81:839-846.

10. Michener, P. M., Pataky, J. K., and White, D. G. 2002. Rates of transmitting Erwinia stewartii from seed to seedlings of a sweet corn hybrid susceptible to Stewart's wilt. Plant Dis. 86:1031-1035.

11. Michener, P. M., Pataky, J. K., and White, D. G. 2002. Transmission of Erwinia stewartii from plants to kernels and reactions of corn hybrids to Stewart's wilt. Plant Dis. 86:167172.

12. Pepper, E. H. 1967. Stewart's Bacterial Wilt of Corn, Monogr. No. 7. American Phytopathological Society, Worcester, MA.

13. Stevens, N. E. 1935. Stewart's disease in relation to winter temperatures. Plant Dis. Rep. 8:141-149.

14. Sutula, C. L., Gillett, J. M., Morrissey, S. M and Ramsdell, D. C. 1986. Interpreting ELISA data and establishing the positive-negative threshold. Plant Dis. 70:722-726. 